\title{
High-Frequency Ultrasound Scattering from Microspheres and Single Cells
}

\author{
RE Baddour
}

University of Toronto

\section{Sherar}

University of Toronto

\section{JW Hunt}

University of Toronto

\section{GJ Czarnota}

University of Toronto

\section{Michael C. Kolios}

Ryerson University

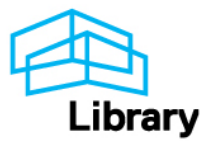




\title{
High-frequency ultrasound scattering from microspheres and single cells
}

\author{
R. E. Baddour, ${ }^{\text {a) }}$ M. D. Sherar, and J. W. Hunt \\ Department of Medical Biophysics, University of Toronto, 610 University Avenue, Toronto, ON, \\ M5G2M9, Canada \\ G. J. Czarnota \\ Department of Radiation Oncology, University of Toronto, 610 University Avenue, Toronto, ON, \\ M5G2M9, Canada \\ M. C. Kolios \\ Department of Mathematics, Physics and Computer Science, Ryerson University, 350 Victoria Street, \\ Toronto, ON, M5B2K3, Canada
}

(Received 2 July 2004; revised 14 October 2004; accepted 20 October 2004)

\begin{abstract}
Assessing the proportion of biological cells in a volume of interest undergoing structural changes, such as cell death, using high-frequency ultrasound (20-100 MHz), requires the development of a theoretical model of scattering by any arbitrary cell ensemble. A prerequisite to building such a model is to know the scattering by a single cell in different states. In this paper, a simple model for the high-frequency acoustic scattering by one cell is proposed. A method for deducing the backscatter transfer function from a single, subresolution scatterer is also devised. Using this method, experimental measurements of backscatter from homogeneous, subresolution polystyrene microspheres and single, viable eukaryotic cells, acquired across a broad, continuous range of frequencies were compared with elastic scattering theory and the proposed cell scattering model, respectively. The resonant features observed in the backscatter transfer function of microspheres were found to correspond accurately to theoretical predictions. Using the spacing of the major spectral peaks in the transfer functions obtained experimentally, it is possible to predict microsphere diameters with less than $4 \%$ error. Such good agreement was not seen between the cell model and the measured backscatter from cells. Possible reasons for this discrepancy are discussed. (C) 2005 Acoustical Society of America. [DOI: 10.1121/1.1830668]
\end{abstract}

PACS numbers: 43.80.Cs, 43.40.Fz, 43.80.Jz, 43.80.Ev [FD] Pages: 934-943

\section{INTRODUCTION}

During the last two decades, several studies have found that localized variations in cell morphologies in tissues (Pavlin et al., 1991; Lockwood et al., 1992) and cell ensembles (Sherar et al., 1987; Berube et al., 1992) can be detected using high-frequency ultrasound from 20 to $100 \mathrm{MHz}$. Although individual cells cannot be resolved, even at the high frequencies used, changes in the brightness and speckle in conventional brightness-mode (B-mode) images as a result of cell structure changes can readily be observed. More recently, spectral analysis techniques (Feleppa et al., 1986; Lizzi et al., 1997) used to analyze radio-frequency (rf) echo signals, have made it possible to more specifically characterize average cell structure changes in tissues (Guittet et al., 1999) and cell ensembles (Kolios et al., 2002, 2003).

Cells undergo many morphological changes throughout the cell cycle, in response to the surrounding environment, and in death. Several types of changes in cell structure have been successfully detected in vitro using high-frequency ultrasound. In particular, changes in structure that occur in cells undergoing mitosis (Czarnota et al., Br. J. Cancer, 1999; Kolios et al., 2002), after exposure to changes in salinity (Czarnota et al., Proc. Microsc. Soc. Am., 1999; Kolios

a)Electronic mail: rbaddour@uhnres.utoronto.ca et al., 2001), during necrotic death (Czarnota et al., 1997), and during apoptosis (Czarnota et al., 1997, 1999, 2002; Kolios et al., 2002) have been shown to induce detectable changes in ultrasound backscatter. Exposure to the drug colchicine, which arrests cells during the metaphase phase of mitosis, increases the ultrasound backscatter of cell ensembles compared to untreated cells. Exposing cells to increased salinity, which induces osmotic cell shrinkage and the condensation of chromatin inside the cell nucleus, also increases the ultrasound backscatter of cell ensembles. In ensembles of necrotic cells (heat killed) and cells undergoing apoptosis (after exposure to cisplatin, a chemotherapeutic agent) an increase in the ultrasound backscattered signal was observed compared to viable cells. Apoptosis, a process marked by cell and nucleus shrinkage, condensation of chromatin in the nucleus, and eventual nuclear fragmentation (Hacker, 2000), causes significant increases in highfrequency ultrasound scattering: up to a 20 -fold change in average backscatter intensity compared to ensembles of viable cells, and possibly an increase in the scattering efficiency at higher frequencies (Kolios et al., 2002).

Although using ultrasound it is possible to noninvasively detect ensembles of cells undergoing structural changes, such as apoptosis, it is difficult to quantify the proportion of cells undergoing these changes. The long-term 
goal of our research is to develop such a quantitative measure for various biological tissues; a potentially useful metric for various applications, such as cancer treatment evaluation. Achieving this goal requires the development of a theoretical model for the scattering by any arbitrary cell ensemble taking into account any packing arrangement and any combination of cell morphologies. A prerequisite for this generalized model is to understand the scattering by a single cell, the building block of any biological tissue. Understanding the scattering response of a single cell will enable the estimation of the properties (e.g., size, shape) and assessment of the functional condition (e.g., viable versus apoptotic) of an arbitrary cell simply by analyzing the ultrasound backscattering from that cell.

Historically, it has not been possible to detect isolated, individual eukaryotic cells even with high-frequency ultrasound devices. The main problems are related to localization and low signal strength. Due to their small size $(5-20 \mu \mathrm{m})$, even at high ultrasound frequencies, cells are smaller than the wavelength of the incident sound wave $(20-75 \mu \mathrm{m})$, the resolution limit for imaging applications. In addition, not only due to their small size, but also their weakly scattering nature, the scattered sound from individual cells has been too low to be detected above background system noise. Very recently, new high-frequency ultrasound devices have emerged with better system signal-to-noise ratio characteristics, which can enable the measure of backscatter from smaller and weaker scatterers. To date, backscattering from individual cells has never been successfully measured. We present a method to deduce the backscattering from individual subresolution scatterers. Using this method, the backscatter from polystyrene microspheres and single cells are measured and used to evaluate a proposed model of the highfrequency acoustic scattering by one living biological cell.

\section{MODEL OF A SINGLE CELL}

In order to calculate the single-cell scattering response, the dominant acoustic scattering centers in cells at high frequencies must be determined. Since apoptosis produces a significant change in the overall backscatter from cell ensembles (Kolios et al., 2002, 2003), it is useful to examine the dominant changes in cells undergoing apoptosis before they break up into apoptotic bodies (a later stage of apoptosis) and get phagocytosed. During the early stages of apoptosis the mitochondria swell, the cell diameter decreases, the nuclear diameter decreases, and the chromatin condenses inside the nucleus. As shown in Fig. 1, most of the significant gross structural changes that occur are related to the nucleus.

The nucleus of a cell is denser than the rest of the cell (Meselson and Stahl, 1958) due to its high DNA and protein content. By estimates, approximately $71 \%$ of the nuclear volume is taken up by chromatin (Monier et al., 2000); chromatin being made up of approximately 50\% DNA and 50\% protein by volume (Kornberg, 1974). Deoxyribonucleic acid has a density of $1.71 \mathrm{~g} / \mathrm{cm}^{3}$ (Meselson and Stahl, 1958), whereas typical proteins have a density of $1.35 \mathrm{~g} / \mathrm{cm}^{3}$ (Matthews, 1968). The rest of the nucleus is a heterogeneous solution of biological macromolecular structures termed the nuclear matrix. By contrast, the composition of the cell

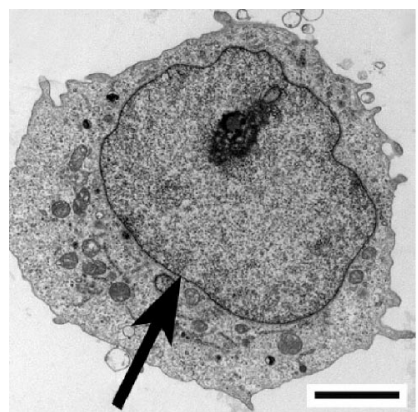

(a)

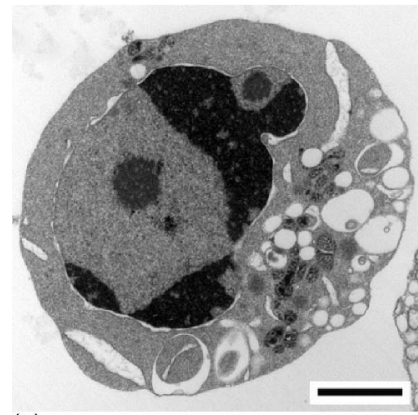

(c)

FIG. 1. Transmission electron micrographs of cells from the OCI-AML-5 line (Wang et al., 1991) (human acute myeloid leukemia) in different stages of apoptosis induced by exposure to cisplatin; scale bars represent $2 \mu \mathrm{m}$. (a) Normal cell; nucleolus and mitochondria are clearly visible, nucleus (indicated by arrow) takes up a large proportion of the cell volume. (b) Early apoptosis; reduction in nuclear diameter and chromatin condensation is evident (nucleus is indicated by arrow); vacuoles and/or swelled mitochondria are visible. (c) Later apoptosis; nuclear fragmentation is starting. (d) Very late apoptosis; apoptotic bodies containing chromatin fragments and other cell constituents.

around the nucleus is mainly cytoplasm, which has a density approximately equal to the surrounding interstitial fluid (a low concentration saline), a small amount of protein (e.g., cytoskeleton), and thin bilipid membranes that make up the other organelles (e.g., Golgi apparatus, mitochondria).

The longitudinal speed of sound in DNA, in varying conformations and orientations, has been measured to be in the range of 1900 to $2400 \mathrm{~m} / \mathrm{s}$ (Hakim et al., 1984; Edwards et al., 1985). This is significantly higher than the speeds of sound of cytoplasm, estimated to be $1508 \mathrm{~m} / \mathrm{s}$ in muscle fiber cells (Berovic et al., 1989), or interstitial fluid, which, due to its high water content, is likely to be close to $1527 \mathrm{~m} / \mathrm{s}$, the value for water at body temperature $\left(37^{\circ} \mathrm{C}\right)$. Therefore, it is reasonable to conclude that the average acoustic impedance of the nucleus is significantly higher than the average acoustic impedance of the rest of the cell and the surrounding fluid. It follows that the nucleus should scatter sound more efficiently than the rest of the cell.

If one assumes that there is not a significant mismatch in density and speed of sound between the balance of the cell around the nucleus and the surrounding medium, it is a reasonable simplification, for acoustic scattering calculations, that a cell could be approximated by its nucleus. In addition, although the nucleus to cytoplasm volume ratio varies by cell type, in many cases the nucleus is relatively isotropic and almost spherical. Therefore, we propose here that a cell be modeled as a single spherical scatterer with uniform me- 


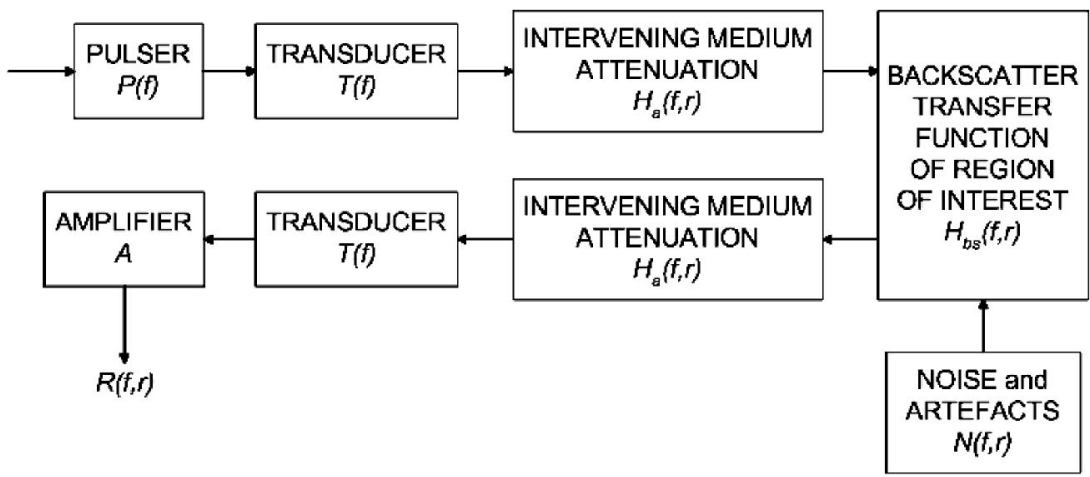

FIG. 2. System block diagram: The pulse, $E(f)$, is generated by the pulser and modified by several transfer functions - transducer, $T(f)$, attenuating medium, $H_{a}(f, r)$, scatterers in the region of interest, $H_{b s}(f, r)$, artifacts and noise, $N(f, r)$ - until the backscattered signal, $R(f, r)$, is finally amplified, by a factor of $A$, and measured.

chanical properties that correspond to its nucleus. Since the proposed cell model is simple geometrically, one can mathematically determine the theoretical scattering response of a cell to incident high-frequency ultrasound pulses by calculating the scattering response of a sphere with nucleus-like mechanical properties.

The far-field complex pressure amplitude $\left(p_{s}\right)$ at an observation point at time $t$ of the scattered echo returned from a nonrigid sphere insonified by a plane, monochromatic acoustic wave can be expressed in series form as

$$
\begin{aligned}
p_{s}\left(t, k_{3}, \theta\right)= & \frac{P_{i} a}{2 r}\left[\frac{2}{x_{3}} \sum_{n=0}^{\infty}(-1)^{n}(2 n+1)\right. \\
& \left.\times \sin \eta_{n} e^{-i \eta_{n}} P_{n}(\cos \theta)\right] e^{-i k_{3}\left(c_{3} t-r\right)},
\end{aligned}
$$

where $P_{i}$ is the incident wave amplitude, $a$ is the radius of the spherical scatterer, $r$ is the distance to the observation point, $c_{3}$ is the longitudinal speed of sound in the surrounding medium, $k_{3}$ is the wave number (for a given frequency $f$, $\left.k_{3}=2 \pi f / c_{3}\right), x_{3}$ is the relative frequency $\left(x_{3}=k_{3} a\right)$, and $P_{n}$ is the $n$ th-order Legendre polynomial of argument $\cos \theta$. The scattering angle $(\theta)$ is equal to $\pi$ in the backscattering case. The phase shift $\eta_{n}$ is a term which takes into account all other parameters, such as the mechanical properties of the scatterer. Faran (1951) developed an expression in the form of Eq. (1) for the scattering by an elastic sphere in water (or any medium that does not support shear), accounting for wave-mode conversion inside the scatterer. A small correction was presented by Hickling (1962), who verified the result experimentally. To calculate theoretical scattered pressures $\left(p_{s}\right)$ for various conditions, the Faran-Hickling solution was used in our studies. Although the developed computer code allows for the calculation of individual scattering conditions, typically the scattered pressures for a range of a particular parameter are calculated at once. For example, to calculate the size dependence of scattering, a range of $a$ values, representing the sphere radii of interest, would be passed to the algorithm along with the wave number $\left(k_{3}\right)$, mechanical properties of the scatterer $\left(c_{1}, \rho_{1}, \sigma\right)$, mechanical properties of the surrounding medium $\left(c_{3}, \rho_{3}\right)$, and the location of the observation point $(\theta, r)$, as defined by Faran (1951). Parametric studies of these properties have been performed by Hickling (1962) and many others (Hampton and McKinney, 1961; Brill and Gaunaurd, 1987; Hinders, 1991).

\section{METHODS}

\section{A. Deducing the backscatter signal from a single subresolution scatterer}

The first step in attempting to measure the backscatter from a single subresolution scatterer was to prepare a sparse suspension of the scatterer in question (microspheres or cells). The ideal suspending medium is a pure liquid, such as water, to eliminate any possibility of medium inhomogeneities (e.g., that would be possible if using a gel). Very low concentrations (between 1000 to 10000 scatterers $/ \mathrm{cm}^{3}$ ) of the scatterers were mixed into degassed water. Preceding every acquisition, the vials containing the suspensions were gently stirred. This stirring step was found to be particularly important for suspensions of larger and denser scatterers due to their higher settling velocities.

Data acquisition was performed using a VisualSonics VS40b (VisualSonics Inc., Canada) ultrasound imaging device which generates short, broadband pulses with center frequencies at 20,30,40, or $55 \mathrm{MHz}$. This device digitally samples the pressure received by the transducer at a rate of $500 \mathrm{MHz}$ with a measurement resolution of 256 levels (8 bits). Several different transducers, with different resonant frequencies, $f$ numbers, and focal lengths, were employed (20 MHz: $f 2.35,20 \mathrm{~mm}$; $30 \mathrm{MHz}: f 2.13,12.75 \mathrm{~mm} ; 40$ MHz: $f 2,6 \mathrm{~mm}$; $55 \mathrm{MHz}: f 3,6 \mathrm{~mm}$ ); each with a $6-\mathrm{dB}$ intensity bandwidth of nearly 100 percent. Raw rf echo signals, the raw A lines, were acquired from different lateral positions with the transducer submerged in water. All of the acquired A lines (roughly 1000 per experiment) were then thresholded, discarding all lines not containing any data value greater than half the maximum data value detected in all the echo signals. This step eliminated the numerous "empty" acquisitions that occur since the suspensions are so sparse, with concentrations equivalent to less than one scatterer per focal volume of the most focused transducer used. In addition, the threshold eliminates most "indirect hits" with scatterers (e.g., a scatterer at the edge of the focal volume or in any sidelobe present in the incident beam profile), which result in backscattered pressure intensities of generally lower peak amplitude, ensuring that only near-pure backscatter signals from single microspheres are analyzed. Spectral analysis was then performed on the 50 to $100 \mathrm{~A}$ lines that remain after thresholding. The part of the signal acquired from around the transducer focus, the depth of field of the transducer (where the incident waves can assumed to be pla- 
nar), was extracted by multiplication with a Hamming window and Fourier transformed to facilitate spectral analysis.

\section{B. The backscatter transfer function}

The experimental ultrasound system was modeled as a function of frequency $(f)$ and location $(r)$. From the system block diagram shown in Fig. 2, Eq. (2) was used to describe the Fourier transform of the signal received from the depth of field, $R(f, r)$

$$
R(f, r)=E(f) T(f)^{2} H_{a}(f, r)^{2}\left[H_{b s}(f, r) \otimes N(f, r)\right] A,
$$

where $E(f)$ is the transmitted electrical pulse, $T(f)$ is the transducer transfer function (a measure of the transducer's efficiency), $H_{a}(f, r)$ is the attenuation transfer function of the intervening medium, $H_{b s}(f, r)$ is the backscatter transfer function of the region of interest (in our case, containing only a single scatterer), $N(f, r)$ is a model of any acoustic artifacts as well as random noise, and $A$ is the amplifier gain. It would be desirable to solve for $H_{b s}(f, r)$ to study the backscatter response of the region of interest. However, $E(f)$, $T(f), H_{a}(f, r)$, and $N(f, r)$ are not known. The solution we adopted was to take a reference measurement, $R_{\text {ref }}(f, r)$ : the perpendicular specular reflection from a flat, polished $\mathrm{SiO}_{2}$ crystal (Edmund Industrial Optics Inc., part 43424; $\rho$ $=2.20 \mathrm{~g} / \mathrm{cm}^{3}, c=5720 \mathrm{~m} / \mathrm{s}$ ) placed at the same depth as the region of interest, normal to the incident pulse direction. $R_{\text {ref }}(f, r)$ will have exactly the same $E(f), T(f)$, and approximately the same $H_{a}(f, r)$ (since scatterer depths will vary inside the region of interest) as $R(f, r) . H_{b s}(f, r)$ for this reference measurement is simply the reflection coefficient $\left[H_{b s}(f, r)=0.79\right.$ in the case of the $\mathrm{SiO}_{2}$ crystal]. As a result, we defined the approximate backscatter transfer function (BSTF) as

$$
\mathrm{BSTF}=\left|\frac{R(f, r)}{R_{\mathrm{ref}}(f, r)}\right|^{2}=\left|\frac{A\left[H_{b s}(f, r) \otimes N(f, r)\right]}{0.79 A_{\text {ref }} N_{\text {ref }}(f, r)}\right|^{2},
$$

which is an approximation of $\left|H_{b s}(f, r)\right|^{2}$. It was more convenient to define the BSTF in terms of squared magnitudes (i.e., power spectra) as they are directly proportional to pressure intensities. The BSTF of a region of interest was expressed in decibels relative $(\mathrm{dBr})$ to the backscatter intensity from the reference. The values of the BSTF for a region containing a single scatterer were directly compared to normalized pressure intensities calculated with the FaranHickling solution.

\section{RESULTS}

\section{A. Polystyrene microspheres}

Before attempting to measure the backscatter from single cells, the method to deduce the backscatter transfer function of a subresolution scatterer was tested with polystyrene microspheres (Beckman Coulter Inc., part numbers 6602796,6602798 , and 6602802 ). These were used because they are homogeneous, have known mechanical properties $(\rho=1.05 \mathrm{~g} / \mathrm{mL}, c=2350 \mathrm{~m} / \mathrm{s}, \sigma=0.35)$, and are available in calibrated sizes (diameter tolerances of $\pm 0.5 \%$ ). The microspheres were suspended in distilled, degassed water at
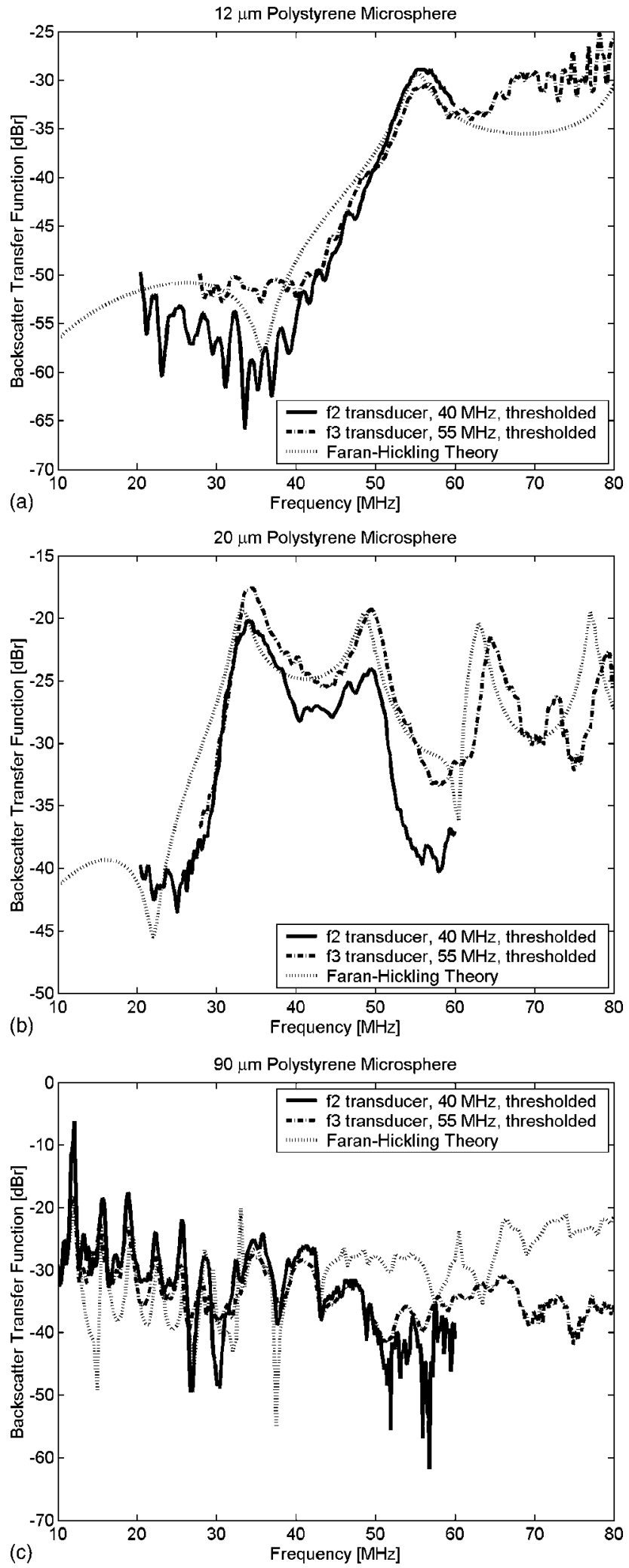

FIG. 3. Theoretical and measured backscatter transfer functions for single (a) $12-\mu \mathrm{m}$; (b) $20-\mu \mathrm{m}$; and (c) $90-\mu \mathrm{m}$ polystyrene microspheres (c $=2350 \mathrm{~m} / \mathrm{s}, \rho=1.05 \mathrm{~g} / \mathrm{mL}, \sigma=0.35$ ) in water using two different wideband transducers (only the data from the 6- $\mathrm{dB}$ bandwidth of each transducer are shown). Note that the theoretical curves have not been shifted or scaled in any way, except to take into account the geometric effect of diminishing solid angle with distance. 


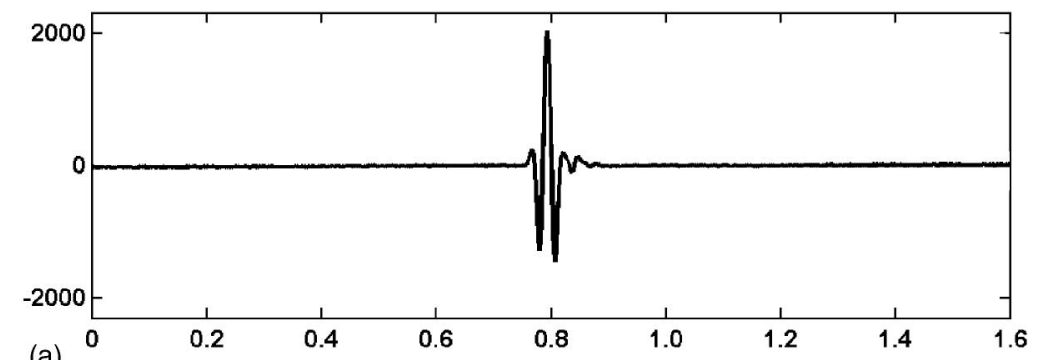

(a)

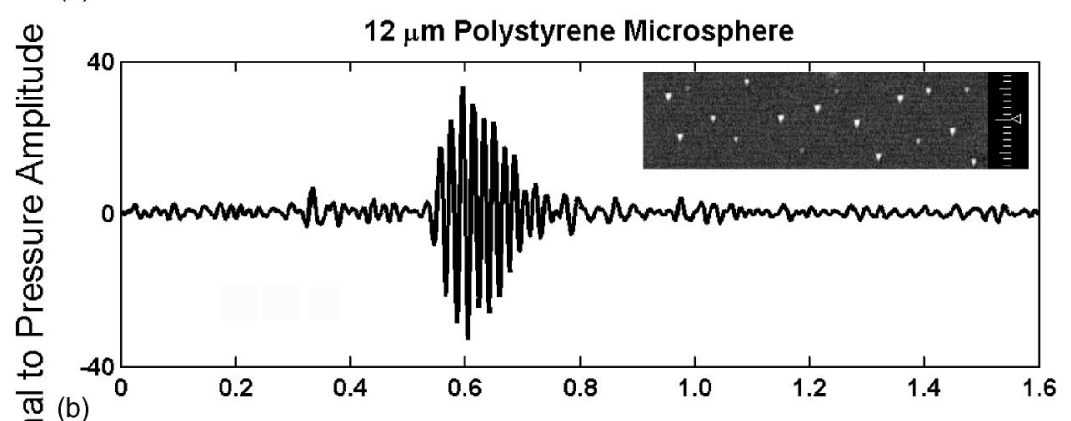

(b)

$20 \mu \mathrm{m}$ Polystyrene Microsphere

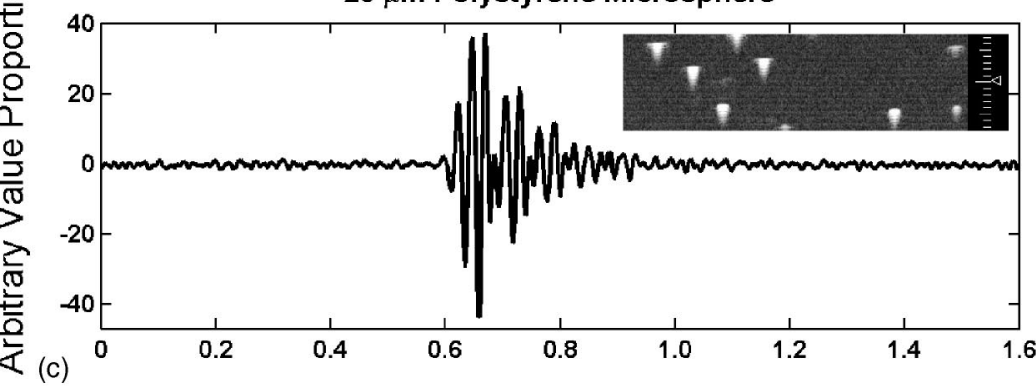

$90 \mu \mathrm{m}$ Polystyrene Microsphere

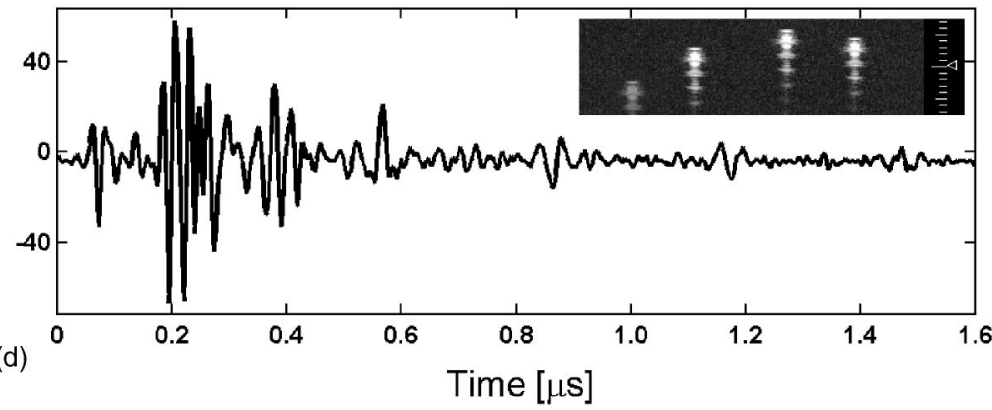

FIG. 4. Typical backscattered pulses from a $12-\mu \mathrm{m}$ (b), $20-\mu \mathrm{m}$ (c), and a 90- $\mu \mathrm{m}$ (d) polystyrene microsphere insonified by an $f 2$ transducer with a broadband 40 $\mathrm{MHz}$ pulse (a). Insets are typical B-scans of sparse suspensions of microspheres (scale resolution $=100 \mu \mathrm{m}$ ); triangles indicate the depth of the transducer focus.

room temperature. Figure 3 shows the measured BSTFs for polystyrene microspheres of three different diameters (12, 20 , and $90 \mu \mathrm{m}$ ) plotted along with the corresponding theoretical normalized backscattered pressure intensity frequency response. Figure 4 shows a representative backscattered pulse measured from each size of microsphere. The smaller sizes were chosen as they are in the range of typical eukaryotic cells $(5-20 \mu \mathrm{m})$. The larger size was chosen to investigate the sensitivity of the experimental setup to detecting the complex high-frequency resonances predicted by the FaranHickling solution.

\section{B. Eukaryotic cells}

Experiments with eukaryotic cells were performed to evaluate the proposed cell scattering model, i.e., an elastic sphere with nucleus-like properties. OCI-AML-5 (Wang et al., 1991), a line of human acute myeloid leukemia cells, was chosen for these experiments. In addition to having a simple morphology with a single, quasispherical nucleus (as seen in Fig. 1), the apoptotic response of these cells has been well documented (Tohda et al., 1996; McCubrey et al., 2001; Saleh et al., 2002), an important feature given one of our ultimate goals of a measure of the apoptotic index. This cell line was also selected because previous high-frequency ultrasound studies have been performed on these cells (Czarnota et al., 1997, 1999; Kolios et al., 2002). Instead of water, the surrounding medium used for the cell suspension was a dilute phosphate buffered saline (PBS) solution (in distilled water: $8 \mathrm{~g} / \mathrm{L}$ sodium chloride, $0.2 \mathrm{~g} / \mathrm{L}$ potassium chloride, $0.132 \mathrm{~g} / \mathrm{L}$ calcium chloride, $0.10 \mathrm{~g} / \mathrm{L}$ magnesium chloride, $1.15 \mathrm{~g} / \mathrm{L}$ sodium phosphate, $0.2 \mathrm{~g} / \mathrm{L}$ potassium phosphate) to 


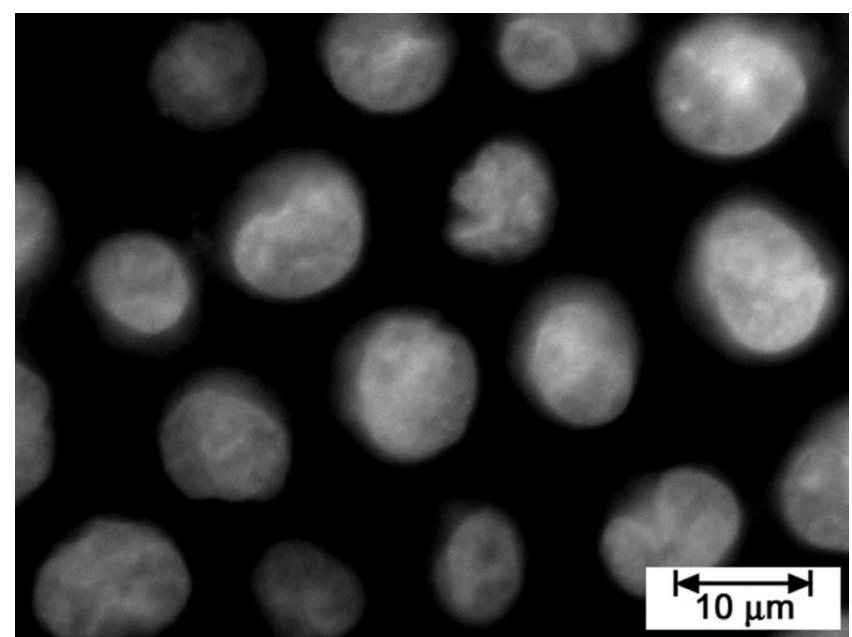

(a)

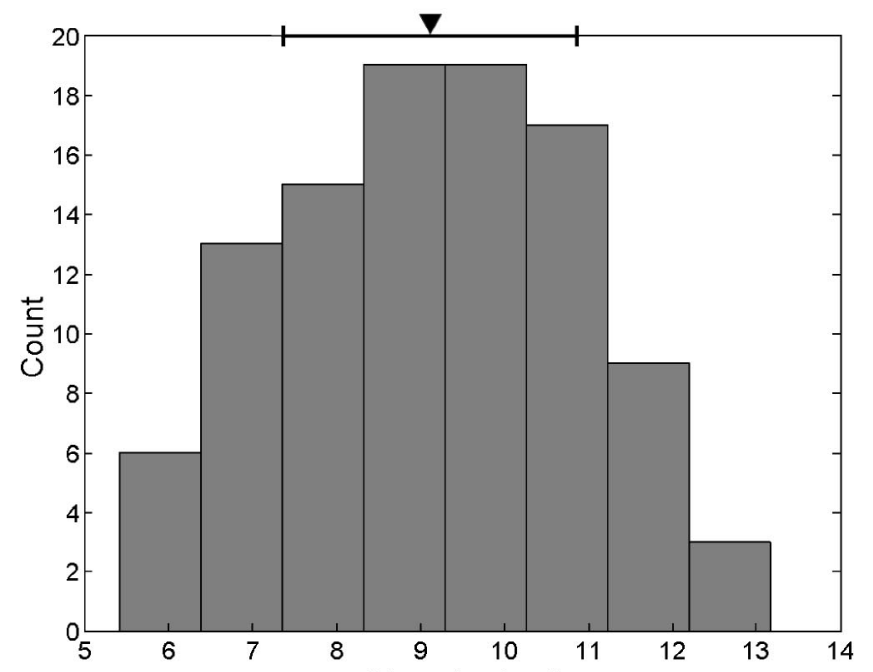

(b)

Diameter $[\mu \mathrm{m}]$

FIG. 5. Measurement of OCI-AML-5 cell nuclei. (a) Optical confocal microscopy of a population of bisbenzimide-stained OCI-AML-5 cells using ultraviolet illumination (only nuclei are visible). (b) Distribution of OCIAML-5 nuclei diameters measured from optical confocal microscopy studies; top scale bar represents $+/-1$ standard deviation centered about the mean (indicated by a triangle). preserve the viability of the cells. This solution of inorganic salts maintains a submerged cell's physiological $p \mathrm{H}$, osmotic equilibrium, and membrane potential.

To calculate the theoretical backscatter from individual OCI-AML-5 cells in PBS using the proposed simplified cell scattering model, the average diameter and mechanical properties of OCI-AML-5 nuclei were required (PBS was assumed to be water-like with $\rho=1.0 \mathrm{~g} / \mathrm{mL}$ and $c$ $=1483 \mathrm{~m} / \mathrm{s}$ ). Using optical confocal microscopy of bisbenzimide-stained cells with ultraviolet illumination, shown in Fig. 5(a), the average diameter of OCI-AML-5 nuclei (of cells from this particular multiply passaged line) was measured to be $9.1 \mu \mathrm{m}$, with a standard deviation of $1.8 \mu \mathrm{m}$. The distribution of measured diameters is presented in Fig. 5(b).

The average longitudinal speed of sound in OCI-AML-5 nuclei, extracted from cells by the method of Muramatsu et al., 1974, was measured to be $c=1508.5 \mathrm{~m} / \mathrm{s}$. This measurement was performed in PBS medium using a dense pellet of nuclei, centrifuged into one of two calibrated wells of a custom-built steel sample holder; the second well was used as a reference. The Poisson's ratio of OCI-AML-5 nuclei from viable cells was assumed to be the same as the Poisson's ratio of nuclei from chondrocytes (connective tissue cells), which has been measured to be $\sigma=0.42$ (Knight et al., 2002).

The average density of an OCI-AML-5 nucleus was estimated assuming generic eukaryotic nucleus properties. Using the estimate that $71 \%$ of the nuclear volume is taken up by chromatin, which contains approximately an equal proportion of DNA and protein by volume, and if one assumes that the rest of the nuclear matrix is an equal combination of dilute brine (essentially water, $\rho=1.0 \mathrm{~g} / \mathrm{mL}$ ) and protein, it is possible to calculate an overall average nucleus density to be $\rho=1.43 \mathrm{~g} / \mathrm{mL}$.

Figure 6 shows the measured BSTF for an OCI-AML-5 cell using three different transducers compared with two corresponding theoretical backscatter pressure intensity curves

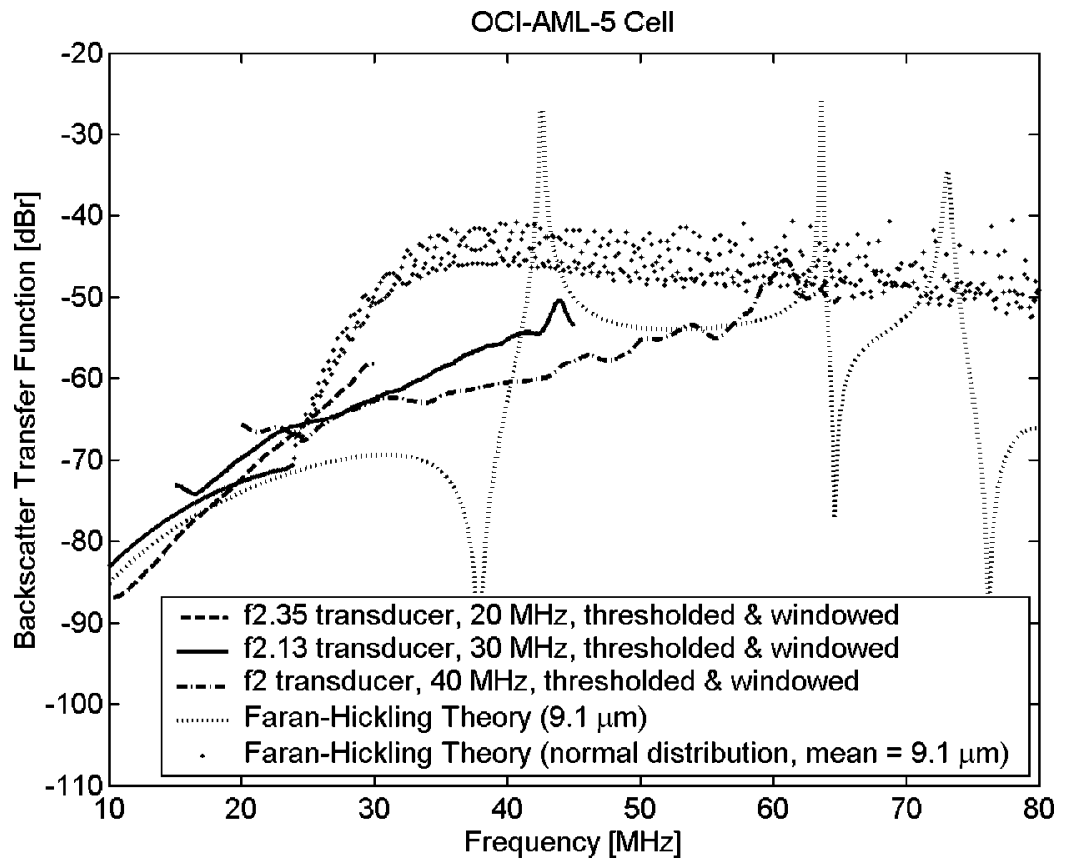

FIG. 6. Theoretical and measured backscatter transfer functions for single OCI-AML-5 cells (theoretical cell model parameters: $c=1508.5 \mathrm{~m} / \mathrm{s}, \rho=1.43 \mathrm{~g} / \mathrm{mL}, \sigma$ $=0.42,2 a=9.1 \mu \mathrm{m}$ ) in PBS (assumed to be water-like for theoretical calculations) using three different wideband transducers (only the data from the 6-dB bandwidth of each transducer are shown). The second theoretical curve (scatter plot) is a weighted average of the Faran-Hickling scattering solutions for a normal distribution of nuclei diameters, corresponding to the distribution of measured diameters (mean $=9.1 \mu \mathrm{m}$, standard deviation $=1.8 \mu \mathrm{m}$ ) as shown in Fig. 5(b). Note that neither theoretical curve was scaled or shifted in any way, except to take into account the geometric effect of diminishing solid angle with distance. 


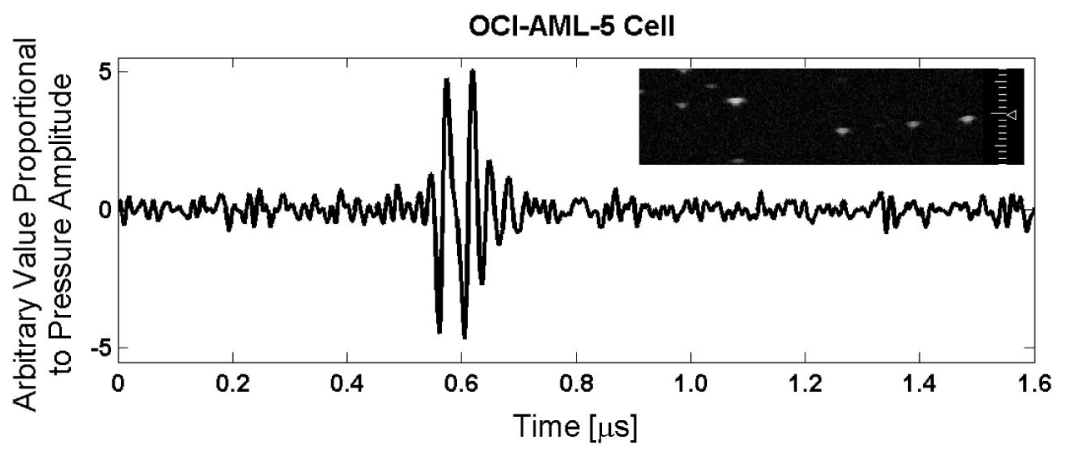

FIG. 7. Typical backscattered pulse from an OCIAML-5 cell insonified by an $f 2$ transducer with a broadband $40-\mathrm{MHz}$ pulse; incident pulse is shown in Fig. 4(a). Inset is a typical B-scan of a sparse suspensions of cells (scale resolution $=100 \mu \mathrm{m}$ ); triangle indicates the depth of the transducer focus.

for spheres with OCI-AML-5 nucleus-like mechanical properties in water. The first theoretical curve (dotted line) is a calculation of the Faran-Hickling scattering solution using the mean measured nucleus diameter of $9.1 \mu \mathrm{m}$. The second theoretical curve (scatter plot) is a weighted average of the Faran-Hickling scattering solutions for a normal distribution of nuclei diameters, corresponding to the distribution of measured diameters $\quad($ mean $=9.1 \mu \mathrm{m}$, standard deviation $=1.8 \mu \mathrm{m}$ ) as shown in Fig. 5(b). Figure 7 shows a representative backscattered pulse measured from an OCI-AML-5 cell.

\section{DISCUSSION AND CONCLUSIONS}

\section{A. Polystyrene microspheres}

Very good agreement in the location of the spectral features predicted by the theory and the experimentally measured data was observed. It was encouraging that even the high-frequency resonances were detected experimentally. However, when the scatterer becomes large, as for the 90- $\mu \mathrm{m}$ microsphere, agreement of feature locations and intensity levels was less rigorous, particularly at high frequencies. This could be due in part to the fact that $90 \mu \mathrm{m}$ is very close to the full-width half-maximum (FWHM) intensity beamwidth of the transducers used. Another likely factor is that the wave components that make up the sharp, peculiarly shaped resonances predicted for $k a>15$ (i.e., $2 \pi f a / c_{3}$ $>15$ ), a range only seen in Fig. 3(c) for frequencies above $40 \mathrm{MHz}$, might disperse (e.g., due to differing propagation speeds per frequency) before reaching the transducer for acquisition. As seen in Fig. 3(c), even when the transducer with a 55-MHz resonant frequency was employed, although there was good agreement at lower frequencies, the agreement was poor near $55 \mathrm{MHz}$ where most of the beam power is centered (and the best results would be expected). This is good evidence that dispersion is occurring and affecting the higher frequencies of scattered sound before they can be measured. The same dispersion should also be present in the results from the 12- and 20- $\mu \mathrm{m}$ microspheres, but as the backscatter responses are less complicatedly shaped in these cases, the effect on agreement with theory is probably less evident.

Some of the features in the backscatter frequency response curves can be interpreted according to resonance theory. If the frequency of the incident wave coincides with one of the resonant modes (vibrational eigenmodes) of the object, a resonant (or ringing) characteristic will be observed in the amplitude of the scattered wave. This behavior, how- ever, is not sufficient to explain the complicatedly shaped curves of Fig. 3 and Fig. 4. Although the sharp peaks at lower frequencies in Fig. 3(c) could well correspond to resonant modes of a spherical $90-\mu \mathrm{m}$ polystyrene microsphere, the complex behavior at higher frequencies is unlikely due to simple resonance.

Based on the observation of long transients by Faran (1951) and on the experimental results of Hickling (1962), another theory has been proposed where the backscatter response is strongly dependent on a train of backscattered echoes (Gaunaurd and Strifors, 1997). Initially the transducer receives a specularly reflected pulse (from the "front" surface of the microsphere) which mirrors the shape of the incident pulse. Then, the transducer receives regularly spaced pulses due to multiply circumnavigating surface wave pulses as proposed by Brill et al. (1981). Their shape deviates somewhat from the incident pulse due to dispersion of the surface waves-since different distances are traveled along the surface depending on the part of the sphere that was initially reached, and their amplitude diminishes with each turn around due to radiative and absorption losses. The shorter the incident pulse, the more distinctly separated are the resulting train of pulses. The spacing should also be related to the longitudinal speed of sound in the medium $\left(c_{3}\right)$, the transverse (shear) speed of sound in the scatterer $\left(c_{2}\right)$, and the diameter of the scatterer $(2 a)$. For a longer incident pulse (or for certain values of $c_{2}, c_{3}$, and $2 a$ ), the train of resulting echo pulses starts to overlap and interference occurs. The type of interference that occurs, constructive or destructive, is dependent on the resonant modes of the scatterer-if the peak of the incident pulse spectrum coincides with a local maximum (a resonant mode) of the theoretical frequency response of the scatterer, then one will get constructive interference; the converse for a minimum. Since the pulses generated by the VisualSonics VS40b device and transmitted by our transducers are broadband (6-dB bandwidths nearly equal to the central frequency), several of these theoretical resonant peaks and troughs are excited at once, so the interference relationship is complicated. For the polystyrene microspheres, we are mainly in this latter regime of overlapping echoes, evident in Fig. 4(b) and Fig. 4(c). For the larger $90-\mu \mathrm{m}$ microsphere, the train of pulses starts to separate; there is some evidence of this phenomenon in Fig. 4(d). This is likely because of the longer time required for the surface waves to circumnavigate the greater circumference of the sphere.

Another important observation from the backscatter re- 


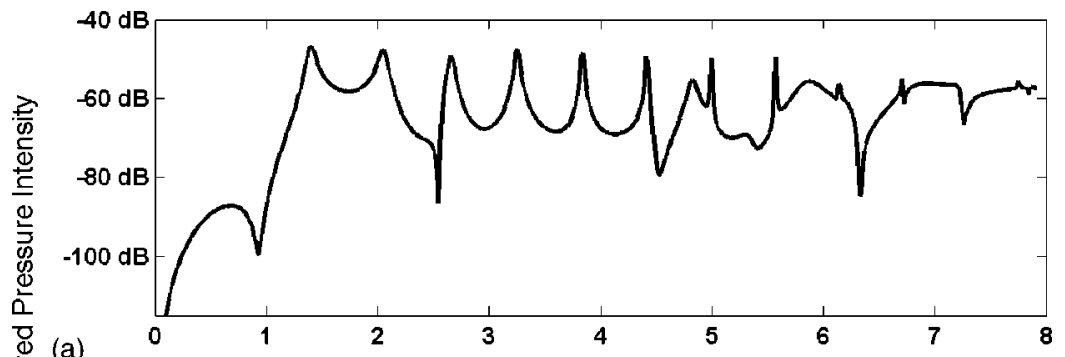

(a)

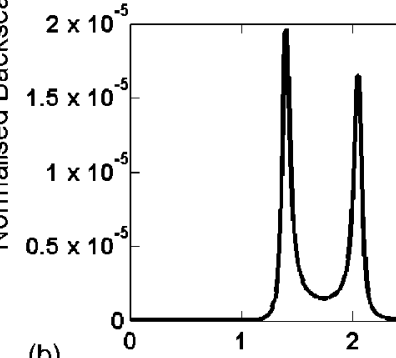

(b)

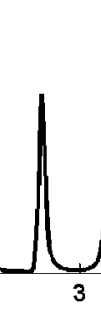

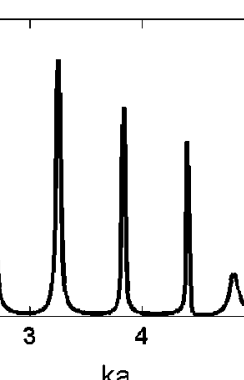

$\mathrm{ka}$
FIG. 8. Theoretical backscatter pressure intensity frequency response for an arbitrarily sized polystyrene microsphere $(c=2350 \mathrm{~m} / \mathrm{s}, \rho=1.05 \mathrm{~g} / \mathrm{mL}, \sigma=0.35)$ plotted on a logarithmic (a) and linear (b) intensity scale (normalized to incident pressure waves of intensity $=1$ ) against the relative frequency, $k a$. sponses of polystyrene microspheres is that the spacing of the resonances in the backscatter frequency response is related to the diameter of the sphere. As the scatterer diameter is decreased the resonances become more distantly spaced. Neglecting the small effect of scatterer size on the backscattered intensity (larger scatterers will naturally reflect more energy than smaller scatterers), the backscatter frequency response for any sphere sharing the same mechanical properties and differing only in size, will be the same curve stretched along the frequency axis by a particular factor related to the sphere diameter. To investigate this phenomenon, the theoretical backscatter response for a polystyrene sphere of an arbitrary diameter in water was calculated and plotted against $k a$, the relative frequency (shown in Fig. 8). Ignoring the absolute intensity values, these curves are valid for any size of polystyrene sphere. From $k a=1$ to $k a=6$, there are very sharp, nearly regularly spaced resonant peaks. The mean spacing between these peaks is $k a=0.6$. Thus, given an experimentally measured backscatter frequency response for a polystyrene sphere, the diameter of the sphere $(2 a)$ could be determined simply from the mean spacing between resonant peaks. For example, in the experimental curves for 20- $\mu \mathrm{m}$ microspheres shown in Fig. 3(b), the average spacing between the resonant peaks is $14.7 \mathrm{MHz}$. Solving for the sphere radius $(a)$

$$
k a=\frac{2 \pi f}{c_{3}} a=\frac{2 \pi\left(14.7 \times 10^{6}\right)}{1483} a=0.6,
$$

yields a predicted diameter of $19.26 \mu \mathrm{m}$; a very good estimate (within 4\%) of the true scatterer size for such a simple calculation. This positive result indicates that the method devised to deduce the backscatter signal from a single subresolution scatterer was successful. The fact that it is possible to work back from a measured backscattered frequency response and deduce characteristics of a subresolution scatterer is quite promising for future work with biological media (if this type of resonant behavior can be observed) for which, in most cases, the nature and properties of the main scattering sources are unknown.

\section{B. Eukaryotic cells}

A typical backscattered pulse from an OCI-AML-5 cell (Fig. 7) was shorter than pulses seen from polystyrene microspheres (Fig. 4). As discussed previously, a shorter scattered pulse is a consequence of a smaller scatterer size. This strong size dependence likely dominates any effect of small relative changes in mechanical properties, such as those between polystyrene, cells, and nuclei. The smallest microsphere diameter studied was $12 \mu \mathrm{m}$, roughly the same diameter as the OCI-AML-5 cells (mean diameter of $13.4 \mu \mathrm{m}$, measured by light microscopy), yet the scattered pulses from cells are shorter than those from these microspheres [Fig. 7 versus Fig. 4(b)]. This adds evidence that the cell nucleus, with its smaller diameter, is probably the main scattering source in the cell. Also, the peak amplitude of the backscattered pulses from cells was lower than those from polystyrene microspheres. This is consistent with the fact that OCIAML-5 nuclei have a lower acoustic impedance $(Z=\rho c$ $\left.=2157 \mathrm{~kg} / \mathrm{m}^{2} \cdot \mathrm{s}\right) \quad$ compared to polystyrene $(Z=\rho c$ $\left.=2468 \mathrm{~kg} / \mathrm{m}^{2} \cdot \mathrm{s}\right)$.

The experimental OCI-AML-5 BSTFs presented in Fig. 6 were approximately continuous across the three different transducers. A trend of reducing slope with increasing frequency is evident. This "corner" may indicate that the band of frequencies investigated represents a transition zone in the scattering response of OCI-AML-5 cells. The large slope of the BSTF from 10 to $30 \mathrm{MHz}$ (this band is mainly covered by the $f 2.35,20-\mathrm{MHz}$ transducer) could indicate the end of the Rayleigh scattering regime. The slope of the $f 2.35$ transducer curve from 10 to $20 \mathrm{MHz}$ is $1.3 \mathrm{~dB} / \mathrm{MHz}$, close to the value of $1.2 \mathrm{~dB} / \mathrm{MHz}$ needed to meet the $f^{4}$ dependence of intensity predicted for Rayleigh scattering. This assessment agrees with the first theoretical curve (Fig. 6 dotted curve), which also predicts higher slopes as frequency decreases. In general, the Faran-Hickling solution predicts that the transi- 
tion zone between the Rayleigh scattering (i.e., higher slope) regime and the more complex resonant scattering regime occurs around $k a=0.5$. In the case of a 9.1- $\mu \mathrm{m}$ scatterer (cell nucleus), this critical value occurs at $26 \mathrm{MHz}$.

It is evident, however, that there is almost no agreement between the first theoretical curve (Fig. 6, dotted curve), the backscattering solution for the mean OCI-AML-5 nucleus diameter, and the experimental results. This is likely due to the wide range of nuclei diameters present in any given sample (as seen in Fig. 5), and consequently in the sparse solutions used in the experiments. As there is no way to easily distinguish between the backscattered pulses when analyzing the results, the calculation of the average BSTF will include the responses from different nucleus sizes (in addition to different shapes, as OCI-AML-5 nuclei are not truly spherical). This will tend to smooth the complicated resonant behavior predicted for the scattering response of a single nucleus (i.e., the local maxima and minima will cancel when shifted along the frequency axis). The second theoretical curve confirms this possibility. The backscatter frequency response of 100 different nuclei sizes (chosen as there are, typically, up to $100 \mathrm{~A}$ lines remaining per experiment after thresholding), normally distributed based on the distribution of measured diameters, each with identical mechanical properties, was calculated using the Faran-Hickling formulation. The weighted sum of these responses (the weights are equal to the probability corresponding to each diameter) is presented in Fig. 6 (scatter plot). As the number of nuclei sizes simulated would increase, this second theoretical curve would become smoother. This theoretical curve agrees more closely with the experimental results, also indicating a "corner-like" response.

Even with a more accurate simulation of the experimental conditions, it is clear that the theoretical backscatter response still does not accurately predict the true response from single OCI-AML-5 cells. It is likely that some of the simplifications made in the theoretical cell model contribute to the divergence between the two results. First, the cell nucleus is not truly spherical [as seen in Fig. 1 and Fig. 5(a)]; it is hard to predict how this would affect the resulting backscatter response as there is no computable solution available for the acoustic scattering from an aspherical, arbitraryshaped elastic scatterer. Another simplification that could potentially have a significant impact on the backscatter from a cell is that the nucleus is not homogeneous. The proposed theoretical model assumes a nucleus with uniform mechanical properties. It is quite possible that small regions of different chromatin density, such as the nucleolus or chromosomes (during mitosis), dominate the resulting scattering process. The high experimental spectral slopes from 10-30 $\mathrm{MHz}$, higher than the theoretical model prediction, also suggest the presence of scatterers of smaller size. Therefore, a nucleus may be better modeled as an ensemble of scatterers.

The assumption that the nucleus by itself is primarily responsible for the acoustic scattering of a cell might also have to be reconsidered. It is likely that there are various other factors affecting the acoustic scattering produced by a single cell. For example, it is possible that the mitochondria, a small organelle with a high density of membranes and pro- teins, could cause a significant amount of scattering. Even more likely, the balance of the cell outside the nucleus likely has some affect on the scattering of incident acoustic pulses, but also on the scattered pulses emitted from the nucleus. This would explain the much lower peak amplitudes of the echo signals measured from nuclei compared to polystyrene microspheres, nearly an eightfold difference, even though the acoustic impedances of each are within $15 \%$. Although cytoplasm, which fills the rest of the cell, is thought to be mostly constituted of water, this is a significant oversimplification biologically. Although 2 to 10 times less stiff than the nucleus (Caille et al., 2002), as a whole, the rest of the cell outside the nucleus is still stiffer than water. This is likely due to one of the important constituents of the cytoplasm, the cytoskeleton (a network of actin, tubulin, myosin, and other proteins), which can significantly change the shape of the cell and its effective resistance to external compressional forces (Caille et al., 2002). There is still much to be learned about the behavior of the cytoskeleton, which varies from one cell type to another and could be modulated by various external or internal cellular stimuli (Karl and Bereiter-Hahn, 1999). It is conceivable that the presence of the mesh of cytoskeleton might dampen, to some unknown extent, the vibrations and transient deformations of the cell and nucleus that would be induced by an incident acoustic pulse. This might, as a result, reduce the amplitude of any morphological resonances in the backscatter frequency response, generally contributing to a smoothing of this curve, as seen in Fig. 6. It might be possible to model the effect of the layer of cytoplasm (containing cytoskeleton) around the nucleus as an elastic shell surrounding a stiffer elastic sphere or a fluid sphere.

Ideally, it would be very helpful at this point to experimentally measure the backscatter of a single OCI-AML-5 cell at various stages of apoptosis and compare it to theoretical predictions using the simplified scattering model of a cell. This would be useful because it would provide a good indication of the model's robustness, which focuses solely on changes to the nucleus, for simulating structural changes such as apoptosis. Practically, however, measuring the backscatter from single cells at specific known stages of apoptosis proved very difficult. We have no method for verifying, by assay or microscopically, that a particular cell being insonified is at a certain stage of apoptosis. This is partly due to the setup, the machine scan head geometry does not allow for concurrent microscopy of the same volume being imaged, but also the thresholding method devised to image single cells. Because cells are in suspension, it is impossible to predict when a cell, let alone a particular cell, will be in the focal zone of the ultrasound beam.

To attempt to make this imaging and backscatter response analysis technique more clinically relevant, experiments will have to be performed using tissues or, in the simplest case, an ensemble of cells. A particular type of ensemble, a pellet of centrifuged cells, is of primary interest given its wide use in preclinical in vitro studies and other ultrasonic studies. In addition to building a more elaborate model to simulate an ensemble, a further complicating factor one might encounter in extending the technique is that the 
major scattering centers may change from the sparse solution (i.e., single-cell) environment, where the balance of the cell surrounding the nucleus appears to have an important effect, to the cellular ensemble condition. For instance, in a tight ensemble of cells, the nuclei may then truly dominate the overall scattering.

\section{ACKNOWLEDGMENTS}

The authors acknowledge Dr. F. S. Foster of the Department of Medical Biophysics at the University of Toronto for intellectual contributions. The authors also acknowledge Anoja Giles, Arthur Worthington, and James Jonkman of the Princess Margaret Hospital for technical support. This work was supported by operating grants from the Canadian Institutes of Health Research and the Whitaker Foundation. The ultrasound imaging device was purchased with funds from the Canadian Foundation for Innovation, the Ontario Innovation Trust, and Ryerson University.

Berovic, N., Thomas, N., Thornhill, R. A., and Vaughan, J. M. (1989). "Observation of Brillouin scattering from single muscle fibers," Eur. Biophys. J. 17, 69-74.

Berube, L. R., Harasiewicz, K., Foster, F. S., Dobrowsky, E., Sherar, M. D., and Rauth, A. M. (1992). "Use of a high-frequency ultrasound microscope to image the action of 2-nitroimidazoles in multicellular spheroids," Br. J. Cancer 65, 633-640.

Brill, D., Gaunaurd, G., and Uberall, H. (1981). "The response-surface in elastic wave scattering," J. Appl. Phys. 52, 3205-3214.

Brill, D., and Gaunaurd, G. C. (1987). "Resonance theory of elastic-waves ultrasonically scattered from an elastic sphere," J. Acoust. Soc. Am. 81, $1-21$.

Caille, N., Thoumine, O., Tardy, Y., and Meister, J. J. (2002). "Contribution of the nucleus to the mechanical properties of endothelial cells," J. Biomech. 35, 177-187.

Czarnota, G. J., Kolios, M. C., Hunt, J. W., and Sherar, M. D. (2002). "Ultrasound imaging of apoptosis: DNA-damage effects visualized," in In Situ Detection of DNA Damage: Methods and Protocols (Methods in Molecular Biology, Vol. 203), edited by V. Didenko (Humana, Totowa, NJ), pp. 257-277.

Czarnota, G. J., Kolios, M. C., Abraham, J., Portnoy, M., Ottensmeyer, F. P., Hunt, J. W., and Sherar, M. D. (1999). "Ultrasound imaging of apoptosis: High-resolution non-invasive monitoring of programmed cell death in vitro, in situ and in vivo," Br. J. Cancer 81, 520-527.

Czarnota, G. J., Kolios, M. C., Vaziri, H., Benchimol, S., Ottensmeyer, F. P., Sherar, M. D., and Hunt, J. W. (1997). "Ultrasonic biomicroscopy of viable, dead and apoptotic cells," Ultrasound Med. Biol. 23, 961-965.

Czarnota, G. J., Kolios, M. C., Heng, Y. M., Deveraj, K., Tam, C., Tan, L., Ottensmeyer, F. P., Hunt, J. W., and Sherar, M. D. (1999). "Ultrasound biomicroscopy of cancer therapy effects: Correlation between light and electron microscopy, and a new noninvasive ultrasound imaging method for detecting apoptosis," Proc. Microsc. Soc. Am. 2320.

Edwards, G. S., Davis, C. C., Saffer, J. D., and Swicord, M. L. (1985). "Microwave-field-driven acoustic modes in DNA," Biophys. J. 47, 799807.

Faran, J. J. (1951). "Sound scattering by solid cylinders and spheres," J. Acoust. Soc. Am. 23, 405-418.

Feleppa, E. J., Lizzi, F. L., Coleman, D. J., and Yaremko, M. M. (1986). "Diagnostic spectrum analysis in ophthalmology-A physical perspective," Ultrasound Med. Biol. 12, 623-631.

Gaunaurd, G. C., and Strifors, H. C. (1997). "Transient resonance scattering and target identification,” Appl. Mech. Rev. 50, 131-148.

Guittet, C., Ossant, F., Vaillant, L., and Berson, M. (1999). "In vivo highfrequency ultrasonic characterization of human dermis," IEEE Trans. Biomed. Eng. 46, 740-746.
Hacker, G. (2000). "The morphology of apoptosis," Cell Tissue Res. 301, 5-17.

Hakim, M. B., Lindsay, S. M., and Powell, J. (1984). "The speed of sound in DNA," Biopolymers 23, 1185-1192.

Hampton, L. D., and McKinney, C. M. (1961). "Experimental study of scattering of acoustic energy from solid metal spheres in water," J. Acoust. Soc. Am. 33, 664-673.

Hickling, R. (1962). "Analysis of echoes from a solid elastic sphere in water," J. Acoust. Soc. Am. 34, 1582-1592.

Hinders, M. K. (1991). "Plane-elastic-wave scattering from an elastic sphere," Nuovo Cimento Soc. Ital. Fis., B 106, 799-818.

Karl, I., and Bereiter-Hahn, J. (1999). "Tension modulates cell surface motility: A scanning acoustic microscopy study," Cell Motil. Cytoskeleton 43, 349-359.

Knight, M. M., Bravenboer, J. V. D. B., Lee, D. A., van Osch, G. J. V. M., Weinans, H., and Bader, D. L. (2002). "Cell and nucleus deformation in compressed chondrocyte-alginate constructs: Temporal changes and calculation of cell modulus," Biochim. Biophys. Acta 1570, 1-8.

Kolios, M. C., Czarnota, G. J., Lee, M., Hunt, J. W., and Sherar, M. D. (2002). "Ultrasonic spectral parameter characterization of apoptosis," U1trasound Med. Biol. 28, 589-597.

Kolios, M. C., Czarnota, G. J., Hussain, M., Foster, F. S., Hunt, J. W., and Sherar, M. D. (2001). "Analysis of ultrasound backscatter from ensembles of cells and isolated nuclei," Proceedings of IEEE Ultrasonics Symposium 1257-1260.

Kolios, M. C., Taggart, L., Baddour, R. E., Foster, F. S., Hunt, J. W., Czarnota, G. J., and Sherar, M. D. (2003). "An investigagion of backscatter power spectra from cells, cell pellets, and microspheres," Proceedings of IEEE Ultrasonics Symposium 752-757

Kornberg, R. D. (1974). "Chromatin structure-Repeating unit of histones and DNA," Science 184, 868-871.

Lizzi, F. L., Astor, M., Feleppa, E. J., Shao, M., and Kalisz, A. (1997). "Statistical framework for ultrasonic spectral parameter imaging," Ultrasound Med. Biol. 23, 1371-1382.

Lockwood, G. R., Ryan, L. K., Gotlieb, A. I., Lonn, E., Hunt, J. W., Liu, P., and Foster, F. S. (1992). "In vitro high-resolution intravascular imaging in muscular and elastic arteries," J. Am. Coll. Cardiol. 20, 153-160.

Matthews, B. W. (1968). "Solvent content of protein crystals," J. Mol. Biol. 33, 491-497.

McCubrey, J. A., Blalock, W. L., Saleh, O. A., Pearce, M., Burrows, C., Steelman, L. S., Lee, J. T., Franklin, R. A., Oberhaus, S. M., Moye, P. W., Doshi, P. D., and McKearn, J. P. (2001). "Enhanced ability of daniplestim and myelopoietin-1 to suppress apoptosis in human hematopoietic cells," Leukemia 15, 1203-1216.

Meselson, M., and Stahl, F. W. (1958). "The replication of DNA in escherichia-coli," Proc. Natl. Acad. Sci. U.S.A. 44, 671-682.

Monier, K., Armas, J. C. G., Etteldorf, S., Ghazal, P., and Sullivan, K. F. (2000). "Annexation of the interchromosomal space during viral infection," Nat. Cell Biol. 2, 661-665.

Muramatsu, M., Hayashi, Y., Onishi, T., Sakai, M., Takai, K., and Kashiyama, T. (1974). "Rapid isolation of nucleoli from detergent purified nuclei of various tumor and tissue-culture cells," Exp. Cell Res. 88, 345351.

Pavlin, C. J., Harasiewicz, K., Sherar, M. D., and Foster, F. S. (1991). "Clinical use of ultrasound biomicroscopy," Ophthalmology 98, 287-295.

Saleh, O. A., Blalock, W. L., Burrows, C., Steelman, L. S., Doshi, P. D., McKearn, J. P., and McCubrey, J. A. (2002). "Enhanced ability of the progenipoietin-1 to suppress apoptosis in human hematopoietic cells," Int. J. Mol. Med. 10, 385-394.

Sherar, M. D., Noss, M. B., and Foster, F. S. (1987). "Ultrasound backscatter microscopy images the internal structure of living tumor spheroids," Nature (London) 330, 493-495.

Tohda, S., Kurokawa, H., and Nara, N. (1996). "Relation of protein kinase a and protein kinase $\mathrm{c}$ to signaling pathways of hematopoietic factors in leukemia cell lines," Int. J. Oncol. 8, 521-524.

Wang, C., Koistinen, P., Yang, G. S., Williams, D. E., Lyman, S. D., Minden, M. D., and McCulloch, E. A. (1991). "Mast-cell growth factor, a ligand for the receptor encoded by c-kit, affects the growth in culture of the blast cells of acute myeloblastic-leukemia," Leukemia 5, 493-499. 